\title{
Determination of Daunomycin in Human Plasma and Urine by Using an Interference-free Analysis of Excitation-Emission Matrix Fluorescence Data with Second-Order Calibration
}

\author{
A-Lin XIA, Hai-Long Wu, ${ }^{\dagger}$ Dong-Mei Fang, Yu-Jie Ding, Le-Qian Hu, and Ru-Qin Yu \\ State Key Laboratory of Chemo/Biosensing and Chemometrics, College of Chemistry and Chemical Engineering, \\ Hunan University, Changsha 410082, People's Republic of China
}

\begin{abstract}
Daunorubicin (DNR) is a significant antineoplastic antibiotic, which is usually applied to a chemotherapy of acute lymphatic and myelogenous leukaemia. Unfortunately, cardiotoxicity research in animals has indicated that DNR is cardiotoxic. Therefore, it is important to quantify DNR in biological fluids. A new algorithm, the alternating fitting residue (AFR) method, and the traditional parallel factor analysis (PARAFAC) have been utilized to directly determine DNR in human plasma and urine. These methodologies fully exploit the second-order advantage of the employed threeway fluorescence data, allowing the analyte concentrations to be quantified even in the presence of unknown fluorescent interferents. Furthermore, in contrast to PARAFAC, more satisfactory results were gained with AFR.
\end{abstract}

(Received November 13, 2005; Accepted May 1, 2006)

\section{Introduction}

Daunorubicin (DNR), discovered in the early 1960s, is an anthracycline antibiotic with antiblastic and anticancer activity, which is linked by the formation of intercalative complexes with DNA and the inhibition of both DNA and RNA synthesis. Although it still remains an important component for many current chemotherapy protocols of acute and myelogenous leukemia, cardiotoxicity is a serious problem, especially in the case of overdoses. ${ }^{1}$ It is thus important for determining DNR in human plasma and urine. Various instrumental methods, such as polarography, ${ }^{2}$ high-performance liquid chromatography, ${ }^{3-5}$ the enzymatic immunological method, ${ }^{6}$ voltammetry, ${ }^{7}$ capillary electrophoresis, ${ }^{8,9}$ and electrospray ionization mass spectrometry ${ }^{10}$ have been reported for DNR determination in biological fluids and in dosage forms. However, these methods are either troublesome or time-consuming.

With the development of modern analytical instrumentation that generate a multidimensional data array for each sample, multiway data arrays have been prominently useful for the quantitative analysis of complex multicomponent samples. Particularly, three-way data arrays following the trilinear model, such as excitation-emission fluorescence matrices, have been gaining widespread analytical acceptance. ${ }^{11}$ Although, interestingly, there are many models for three-way data arrays, only the PARAFAC model ensures uniqueness in a straightforward way. ${ }^{12}$ In addition, the decomposition of a three-way data array built with response matrices measured for many samples is often unique, allowing relative concentrations and spectral profiles of individual sample components to be extracted directly. That is, the analysis of several components of interest can be quantified even in the presence of unknown

$\doteqdot$ To whom correspondence should be addressed. E-mail: hlwu@hnu.cn interferents, commonly called the "second-order advantage". 13,14 The different multiway methods used to resolve multicomponent mixtures belong to three main groups. The first group is direct solution. It is to resolve the data arrays by utilizing an eigenanalysis-based procedure, which typically works well when the signal-to-noise ratio is high. The generalized rank annihilation method (GRAM) $)^{15-17}$ and the direct trilinear decomposition (DTLD) method ${ }^{18-20}$ are wellknown examples. Unfortunately, GRAM is constrained to use only one standard and one mixture sample at a time. Although the DTLD method takes into account a direct solution through multiple samples, it is required to construct two pseudosamples, which unavoidably results in the loss of information in multiple samples. In addition, these methods may occasionally produce imaginary solutions and exhibit inflated variance. The second main group includes least-squares methods. Bilinear least squares (BLLS) is a recently introduced technique, based on a direct least-squares procedure. ${ }^{21,22}$ The third main group is an iterative one. ${ }^{12,23-40}$ Iterative algorithms have been widely employed. The PARAFAC algorithm proposed by Harshman ${ }^{34}$ is a representative method of the third group. These have successfully solved many practical problems, such as environmental monitoring, ${ }^{41}$ the analysis of biological samples, ${ }^{42,43}$ and the quantitation of drugs. ${ }^{44}$ Several algorithms ${ }^{18,25,35-40}$ proposed recently were compared by Faber $e t$ $a l .{ }^{45}$ Their results showed that the developments of three-way data analysis and second-order calibration are still indispensable.

In the present work, an alternating fitting residue (AFR) method was developed for the trilinear analysis of excitationemission matrix fluorescence data. The results revealed that the determination of DNR can be conveniently achieved in plasma and urine samples by second-order fluorescence measurements, and that the performance of the newly introduced AFR method is similar or equal to that of PARAFAC. 


\section{Nomenclature}

Throughout this paper, scalars are represented by lower-case italics; bold lower-case characters mean vectors; bold capitals designate two-way matrices; underlined bold capitals symbolize three-way arrays, and the superscript $\mathrm{T}$ denotes the transpose of a matrix. Before reading the following parts of this paper, readers are recommended to refer the nomenclature for detailed information. The details are as follows: $\underline{\mathbf{X}}$, three-way data array; $I, J, K$, the dimensions of three modes of $\underline{\mathbf{X}} ; \mathbf{A}_{\mathrm{I} \times \mathrm{N}}, \mathbf{B}_{\mathrm{J} \times \mathrm{N}}$, $\mathbf{C}_{\mathrm{K} \times \mathrm{N}}$, the three underlying loading matrices of $\underline{\mathbf{X}}$ with $I \times N, J \times$ $N, K \times N$, respectively (which will be simply represented by $\mathbf{A}$, $\mathbf{B}$ and $\mathbf{C}$, respectively, in this paper); $\mathrm{x}_{\mathrm{ijk}}$, the $i j \mathrm{kth}$ element of $\underline{\mathbf{X}}$; $\mathrm{a}_{\mathrm{in}}, \mathrm{b}_{\mathrm{jn}}, \mathrm{c}_{\mathrm{kn}}$, the inth, jnth and knth elements of the three underlying loading matrices $\mathbf{A}, \mathbf{B}$ and $\mathbf{C}$, respectively; $\mathrm{a}_{(\mathrm{i})}, \mathrm{b}_{(\mathrm{j})}$, $\mathrm{c}_{(\mathrm{k})}$, the $i$ th, $j$ th, $k$ th row vectors of profile matrices $\mathbf{A}, \mathbf{B}$ and $\mathbf{C}$, respectively; $\operatorname{diag}\left(\mathrm{a}_{(\mathrm{i})}\right), \operatorname{diag}\left(\mathrm{b}_{(\mathrm{j})}\right), \operatorname{diag}\left(\mathrm{c}_{(\mathrm{k})}\right)$, diagonal matrices with elements equal to the elements of $\mathrm{a}_{(\mathrm{i})}, \mathrm{b}_{(\mathrm{j})}$ and $\mathrm{c}_{(\mathrm{k})}$, respectively; $\mathbf{X}_{\mathrm{i} . .}, \mathbf{X}_{\mathrm{j} .}, \mathbf{X}_{. \mathrm{k}}$, the $i$ th horizontal, $j$ th lateral and $k$ th frontal slices of $\underline{\mathbf{X}}$ respectively; $\mathbf{E}_{\mathrm{i} . .}, \mathbf{E}_{\mathrm{j} .}, \mathbf{E}_{. \mathrm{k}}$, the $i$ th horizontal, $j$ th lateral and $k$ th frontal slices of the three-way array residue $\underline{\mathbf{E}}$, respectively; $e_{\mathrm{ijk}}$, the $i j k$ th element of the three-way residue array $\underline{\mathbf{E}} ; \mathbf{A}^{+}, \mathbf{B}^{+}, \mathbf{C}^{+}$, the Moore-Penrose generalized inverse of matrices $\mathbf{A}, \mathbf{B}$ and $\mathbf{C}$, respectively; $\|\cdot\|_{\mathrm{F}}$, the Frobenius matrix norm.

\section{Theory}

\section{Trilinear model for second-order calibration}

In the second-order calibration, the famous trilinear model proposed by Harshman ${ }^{34}$ and Carroll and Chang ${ }^{35}$ has been independently widely accepted, owing to its consistency with Beer's law in chemistry. According to the trilinear model, each element $\mathrm{x}_{\mathrm{ijk}}$ of the data array $\underline{\mathbf{X}}$ can be represented as follows:

$$
\mathrm{x}_{\mathrm{ijk}}=\sum_{n=1}^{N} \mathrm{a}_{\mathrm{in}} \mathrm{b}_{\mathrm{jn}} \mathrm{c}_{\mathrm{kn}}+e_{\mathrm{ijk}}, i=1,2, \cdots, I ; j=1,2, \cdots, J ; k=1,2, \cdots, K .
$$

The trilinear model can be expressed as three fully equivalent matrix forms owing to the cyclic symmetry of model, ${ }^{25}$ as follows:

$$
\begin{aligned}
& \mathbf{X}_{\mathrm{i..}}=\mathbf{B} \operatorname{diag}\left(\mathrm{a}_{(\mathrm{i})}\right) \mathbf{C}^{\mathrm{T}}+\mathbf{E}_{\mathrm{i..},}, \quad i=1,2, \cdots, I \\
& \mathbf{X}_{\mathrm{j} .}=\operatorname{Cdiag}\left(\mathrm{b}_{(\mathrm{j})}\right) \mathbf{A}^{\mathrm{T}}+\mathbf{E}_{\mathrm{j} .,}, \quad j=1,2, \cdots, J \\
& \mathbf{X}_{. . \mathrm{k}}=\operatorname{Adiag}\left(\mathrm{c}_{(\mathrm{k})}\right) \mathbf{B}^{\mathrm{T}}+\mathbf{E}_{. . \mathrm{k}}, \quad k=1,2, \cdots, K
\end{aligned}
$$

Regardless of scaling and permutation, the decomposition of the trilinear model proposed above will be a unique one, and no free rotations occur provided that $k_{1}+k_{2}+k_{3} \geqslant 2 N+2,{ }^{46}$ where $k_{1}, k_{2}$ and $k_{3}$ are the $k$-ranks of $\mathbf{A}, \mathbf{B}$ and $\mathbf{C}$, respectively. In the words, the loading matrices $\mathbf{A}, \mathbf{B}$ and $\mathbf{C}$ will be obtained in a unique way.

\section{Least squares-based AF error}

For a trilinear model $\mathbf{X}_{\mathrm{i} . .}=\mathbf{B} \operatorname{diag}\left(\mathrm{a}_{(\mathrm{i})}\right) \mathbf{C}^{\mathrm{T}}+\mathbf{E}_{\mathrm{i} . . .}(i=1,2, \cdots, I)$, one can obtain the following new residues:

$$
\begin{aligned}
& \mathbf{B}^{+} \mathbf{E}_{\mathrm{i} . .}=\mathbf{B}^{+} \mathbf{X}_{\mathrm{i} . .}-\mathbf{B}^{+} \mathbf{B} \operatorname{diag}\left(\mathrm{a}_{(\mathrm{i})}\right) \mathbf{C}^{\mathrm{T}}, \quad i=1,2, \cdots, I \\
& \mathbf{E}_{\mathrm{i} . .}\left(\mathbf{C}^{\mathrm{T}}\right)^{+}=\mathbf{X}_{\mathrm{i} . .}\left(\mathbf{C}^{\mathrm{T}}\right)^{+}-\mathbf{B} \operatorname{diag}\left(\mathrm{a}_{(\mathrm{i})}\right) \mathbf{C}^{\mathrm{T}}\left(\mathbf{C}^{\mathrm{T}}\right)^{+}, \quad i=1,2, \cdots, I
\end{aligned}
$$

Similarly,

$$
\begin{aligned}
& \mathbf{C}^{+} \mathbf{E}_{\mathrm{j} .}=\mathbf{C}^{+} \mathbf{X}_{\mathrm{j} .}-\mathbf{C}^{+} \mathbf{C d i a g}\left(\mathrm{b}_{(\mathrm{j})}\right) \mathbf{A}^{\mathrm{T}}, \quad j=1,2, \cdots, J \\
& \mathbf{E}_{\mathrm{j} .}\left(\mathbf{A}^{\mathrm{T}}\right)^{+}=\mathbf{X}_{\mathrm{j} .}\left(\mathbf{A}^{\mathrm{T}}\right)^{+}-\mathbf{C d i a g}\left(\mathrm{b}_{\mathrm{j})}\right) \mathbf{A}^{\mathrm{T}}\left(\mathbf{A}^{\mathrm{T}}\right)^{+}, \quad j=1,2, \cdots, J \\
& \mathbf{A}^{+} \mathbf{E}_{. . \mathrm{k}}=\mathbf{A}^{+} \mathbf{X}_{. \mathrm{k}}-\mathbf{A}^{+} \mathbf{A} \operatorname{diag}\left(\mathrm{c}_{(\mathrm{k})}\right) \mathbf{B}^{\mathrm{T}}, \quad k=1,2, \cdots, K \\
& \mathbf{E}_{. . \mathrm{k}}\left(\mathbf{B}^{\mathrm{T}}\right)^{+}=\mathbf{X}_{. \mathrm{k}}\left(\mathbf{B}^{\mathrm{T}}\right)^{+}-\mathbf{A} \operatorname{diag}\left(\mathrm{c}_{(\mathrm{k})}\right) \mathbf{B}^{\mathrm{T}}\left(\mathbf{B}^{\mathrm{T}}\right)^{+}, \quad k=1,2, \cdots, K
\end{aligned}
$$

The AFR method makes use of above residues, respectively, to construct three objective functions. One then decomposes the model by alternately minimizing the following three objective functions (AF error):

$$
\begin{aligned}
& \mathrm{S}(\mathrm{C})=\sum_{i=1}^{I}\left\|\mathbf{B}^{+} \mathbf{X}_{\mathrm{i} . .}-\mathbf{B}^{+} \mathbf{B} \operatorname{diag}\left(\mathrm{a}_{(\mathrm{i})}\right) \mathbf{C}^{\mathrm{T}}\right\|^{2}{ }_{\mathrm{F}} \\
& +p \sum_{j=1}^{J}\left\|\mathbf{X}_{\mathrm{j} .}\left(\mathbf{A}^{\mathrm{T}}\right)^{+}-\mathbf{C d i a g}\left(\mathrm{b}_{(\mathrm{j})}\right) \mathbf{A}^{\mathrm{T}}\left(\mathbf{A}^{\mathrm{T}}\right)^{+}\right\|^{2} \mathrm{~F}, \\
& \mathbf{S}(\mathrm{A})=\sum_{j=1}^{J}\left\|\mathbf{C}^{+} \mathbf{X}_{\mathrm{j} .}-\mathbf{C}^{+} \mathbf{C d i a g}\left(\mathrm{b}_{(\mathrm{j})}\right) \mathbf{A}^{\mathrm{T}}\right\|^{2}{ }_{\mathrm{F}} \\
& +q \sum_{k=1}^{K}\left\|\mathbf{X}_{. k}\left(\mathbf{B}^{\mathrm{T}}\right)^{+}-\operatorname{Adiag}\left(\mathbf{c}_{(\mathrm{k})}\right) \mathbf{B}^{\mathrm{T}}\left(\mathbf{B}^{\mathrm{T}}\right)^{+}\right\|^{2}, \\
& \mathrm{~S}(\mathrm{~B})=\sum_{k=1}^{K}\left\|\mathbf{A}^{+} \mathbf{X}_{. \mathrm{k}}-\mathbf{B}^{+} \mathbf{B} \operatorname{diag}\left(\mathrm{c}_{(\mathrm{k})}\right) \mathbf{B}^{\mathrm{T}}\right\|^{2}{ }_{\mathrm{F}} \\
& +r \sum_{i=1}^{I}\left\|\mathbf{X}_{\mathrm{i} . . .}\left(\mathbf{C}^{\mathrm{T}}\right)^{+}-\mathbf{B} \operatorname{diag}\left(\mathrm{a}_{(\mathrm{i})}\right) \mathbf{C}^{\mathrm{T}}\left(\mathbf{C}^{\mathrm{T}}\right)^{+}\right\|_{\mathrm{F}}^{2},
\end{aligned}
$$

where $p, q$ and $r$ are the fitting factors. The function of the fitting factors is to optimize the model for different chemical systems. Generally, we can choose $p=q=r=1$.

\section{The AFR algorithm}

According to the above-mentioned objective functions, an alternating fitting algorithm is used to exploit the solution. That is, one minimizes in an alternating manner the AF error Eq. (11) over $\mathbf{C}$ for fixed $\mathbf{A}$ and $\mathbf{B}$, Eq. (12) over $\mathbf{A}$ for fixed $\mathbf{B}$ and $\mathbf{C}$ and Eq. (13) over $\mathbf{B}$ for fixed $\mathbf{A}$ and $\mathbf{C}$. Since $\mathbf{A}, \mathbf{B}$ and $\mathbf{C}$ can commonly satisfy full column rank, one can obtain three equations, simplified as follows:

$$
\begin{gathered}
\mathbf{C}=\left[\sum_{i=1}^{I} \mathbf{X}_{\mathrm{i..}}{ }^{\mathrm{T}}\left(\mathbf{B}^{+}\right)^{\mathrm{T}} \operatorname{diag}\left(\mathrm{a}_{(\mathrm{i})}\right)+p \sum_{j=1}^{J} \mathbf{X}_{\mathrm{j} .}\left(\mathbf{A}^{\mathrm{T}}\right)^{+} \operatorname{diag}\left(\mathrm{b}_{(\mathrm{j})}\right)\right] \times \\
{\left[\sum_{i=1}^{I}\left(\operatorname{diag}\left(\mathrm{a}_{(\mathrm{i})}\right)\right)^{2}+p \sum_{j=1}^{J}\left(\operatorname{diag}\left(\mathrm{b}_{(\mathrm{j})}\right)\right)^{2}\right]^{+},} \\
\left.\mathbf{A}=\left[\sum_{j=1}^{J} \mathbf{X}_{\mathrm{j} .}^{\mathrm{T}}\left(\mathbf{C}^{+}\right)^{\mathrm{T}} \operatorname{diag}\left(\mathrm{b}_{(\mathrm{j})}\right)\right]+q \sum_{k=1}^{K} \mathbf{X}_{\mathrm{k}}\left(\mathbf{B}^{\mathrm{T}}\right)^{+} \operatorname{diag}\left(\mathrm{c}_{(\mathrm{k})}\right)\right] \times \\
{\left[\sum_{j=1}^{J}\left(\operatorname{diag}\left(\mathrm{b}_{(\mathrm{j})}\right)\right)^{2}+q \sum_{k=1}^{K}\left(\operatorname{diag}\left(\mathrm{c}_{(\mathrm{k})}\right)\right)^{2}\right]^{+}}
\end{gathered}
$$

and

$$
\begin{gathered}
\mathbf{B}=\left[\sum_{k=1}^{K} \mathbf{X}_{. . \mathrm{k}}^{\mathrm{T}}\left(\mathbf{A}^{+}\right)^{\mathrm{T}} \operatorname{diag}\left(\mathrm{c}_{(\mathrm{k})}\right)+r \sum_{i=1}^{I} \mathbf{X}_{\mathrm{i} . .}\left(\mathbf{C}^{\mathrm{T}}\right)^{+} \operatorname{diag}\left(\mathrm{a}_{(\mathrm{i})}\right)\right] \times \\
{\left[\sum_{i=1}^{I}\left(\operatorname{diag}\left(\mathrm{a}_{(\mathrm{i})}\right)\right)^{2}+r \sum_{k=1}^{K}\left(\operatorname{diag}\left(\mathrm{c}_{(\mathrm{k})}\right)^{2}\right)\right]^{+} .}
\end{gathered}
$$

Having mentioned the updating equations for these parameter matrices, we can present the general algorithm for the AFR method as follows:

1. Randomly initialize $\mathbf{A}$ and $\mathbf{B}$, and choose three appropriate fitting factors $p, q, r$. In this paper, we choose $p=q=r=1$.

2. Compute $\mathbf{C}$ using Eq. (14).

3. Compute A using Eq. (15). 
4. Compute B using Eq. (16).

5. Scale $\mathbf{A}$ and $\mathbf{B}$ to be columnwise normalized.

6. Compute $\mathbf{C}$ using Eq. (14).

7. Repeat steps 3 to 6 until a stopping criterion is satisfied. For prediction, see Ref. 25.

The number of responsive factors $(N)$ can be estimated by several methods. A useful technique is to consider the PARAFAC model internal parameter, known as core consistency; typically, the latter one is computed for a number of trial components, and $N$ is set as one less the number for which the core consistency value drops from 100 to less than $50 .{ }^{47}$ A PARAFAC model constructed with the correct number of components is considered to be correct if reasonably low values of the least-squares errors are obtained compared with the instrumental noise level.

\section{Figures of Merit}

Figures of merit are frequently employed for comparing methods. They are best understood by resorting to the useful concept of net analyte signal (NAS), first developed by Lorber. ${ }^{48}$ Different approaches have been discussed in the literature for computing the figures of merit for higher-order methodologies. ${ }^{49-52}$

As for the sensitivity (SEN), the following equation seems to apply to the presently studied case: ${ }^{53-55}$

$$
\mathrm{SEN}=k\left\{\left[\left(\mathbf{A}^{\mathrm{T}} \mathbf{A}\right) *\left(\mathbf{B}^{\mathrm{T}} \mathbf{B}\right)\right]^{-1}\right\}_{\mathrm{nn}}{ }^{-1 / 2},
$$

where $n n$ designates the $(n, n)$ element of a matrix, $k$ is the total signal for component $n$ at unit concentration, and $*$ denotes the Hadamard product. In this paper, $k$ is also the parameter converting scores to concentrations. Notice that the full sensitivities decrease in the presence of other sample constituents, by a degree that depends on the profile overlapping.

The selectivity (SEL), in turn, is the ratio between the sensitivity and the total signal, and can be estimated as ${ }^{53-55}$

$$
\mathrm{SEL}=\left\{\left[\left(\mathbf{A}^{\mathrm{T}} \mathbf{A}\right)^{*}\left(\mathbf{B}^{\mathrm{T}} \mathbf{B}\right)\right]^{-1}\right\}_{\mathrm{nn}}^{-1 / 2} .
$$

Note that when the second-order advantage is employed, Eqs. (17) and (18) imply that SEN and SEL are sample-specific and cannot be defined for the multivariate method as a whole. In such cases, average values for a set of samples can be estimated and reported.

The limit of detection (LOD) $)^{56,57}$ is calculated as

$$
\mathrm{LOD}=3.3 s(0)
$$

where $s(0)$ is the standard deviation in the concentration estimated for three different background blank samples, in the PARAFAC and AFR.

\section{Experimental}

\section{Apparatus}

All of fluorometric measurements were carried out on an F4500 fluorescence spectrophotometer (Hitachi) fitted with a Xenon lamp and interfaced to a 80486 personal computer. In all cases, a $1.00 \mathrm{~cm}$ quartz cell was used. All computer programs were in-house written in Matlab, and all calculations were carried out on a personal computer Pentium IV processor with
Table 1 DNR concentrations $\left(\mu \mathrm{g} \mathrm{ml}^{-1}\right)$ of samples No. 1 - 6

\begin{tabular}{lcccccc}
\hline Sample & 1 & 2 & 3 & 4 & 5 & 6 \\
DNR & 0.44 & 0.66 & 0.88 & 1.32 & 1.54 & 1.76 \\
\hline
\end{tabular}

256 MB RAM under the Windows XP operating system.

\section{Reagents and solution}

DNR was purchased from Sigma-Aldrich (India). A stock solution with a concentration of $22 \mu \mathrm{g} \mathrm{ml}^{-1}$ was prepared by dissolving the DNR in water and stored in glass at $4^{\circ} \mathrm{C}$, protected from light, for a maximum period of one week. Deionized water (Millipore) was used in all experimental preparations. A phosphate buffer solution of $\mathrm{pH} 7.0$ was prepared. The human plasma was purchased from the Blood Center in Changsha. The urine was taken from a healthy individual.

\section{Analytical methodology}

DNR is usually injected as a dose of $45 \mathrm{mg} \mathrm{m}^{-2}$, giving an initial concentration of about $15 \mu \mathrm{g} \mathrm{ml}^{-1}$ in an adult male. Only a fraction of this injected drug will be present in the plasma. In addition, $5-15 \%$ of the drug injected is excreted with urine. Therefore, we decided to work in concentrations not superior to $50 \%$ for plasma and $15 \%$ for urine of the initial dose. The first six samples only contained DNR and were used as concentration calibration samples. The concentrations are given in Table 1. Samples No. 7-11 contained not only DNR, but also human plasma diluted with water (1:5). The last four included DNR and urine diluted with water (1:4). All samples contained a phosphate buffer solution ( $\mathrm{pH}$ 7.0). The excitationemission fluorescence spectra were recorded at excitation wavelengths from 413 to $527 \mathrm{~nm}$ at regular steps of $3 \mathrm{~nm}$ and the emission wavelengths from 533 to $650 \mathrm{~nm}$ at $3 \mathrm{~nm}$ steps. The slit width was $5.0 / 5.0 \mathrm{~nm}$. The scan rate was $1200 \mathrm{~nm} / \mathrm{min}$. For each sample a matrix of fluorescence intensities with dimensions $41 \times 41$ (excitation wavelengths $\times$ emission wavelengths) was obtained.

\section{Results and Discussion}

\section{Core consistency diagnostic (CORCONDIA)}

All data sets were utilized for the core consistency evaluation, using one to four factors. While the core consistency values are negative, they are caused to equal zeros. The results are also shown in Fig. 1. The analysis using CORCONDIA based on the traditional PARAFAC or the AFR algorithms indicates that two factors are necessary for plasma samples, because the utilization of more factors leads to a great decrease in the core consistency, and hence in the trilinearity of the data modeled. A possible explication for these results is that the chemical species in plasma samples is very similar, and PARAFAC and AFR did not resolve these into separated factors and considered one factor. ${ }^{44}$ In addition, two factors are acquired using CORCONDIA based on PARAFAC for urine samples. However, three factors may also be chosen for AFR, because the AFR can solve nonlinear constituents by fitting more factors.

\section{Plasma and urine sample results}

The results obtained for spiked plasma and urine samples clearly illustrate the meaning and usefulness of the second-order 

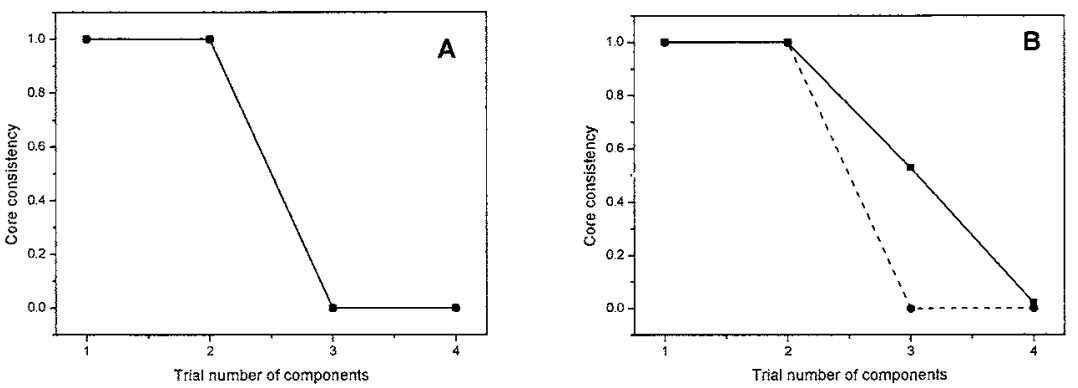

Fig. 1 Core consistency values based on the traditional PARAFAC ( - ) and AFR (- - $)$ algorithms as a function of the trial number of component for the analysis of the spiked plasma (A) and urine $(\mathrm{B})$.
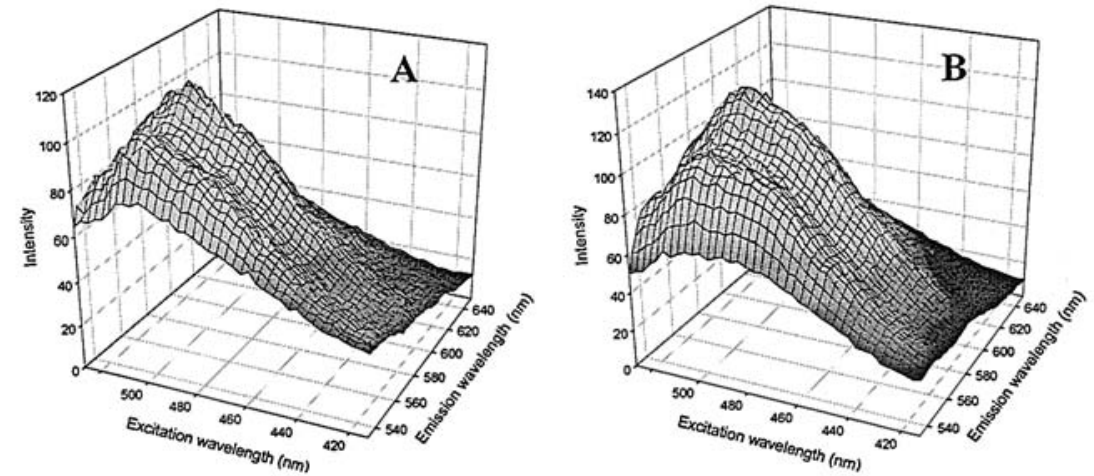

Fig. 2 Three-dimensional plots of the excitation-emission matrix fluorescence for sample 8 (A) and sample 12 (B).
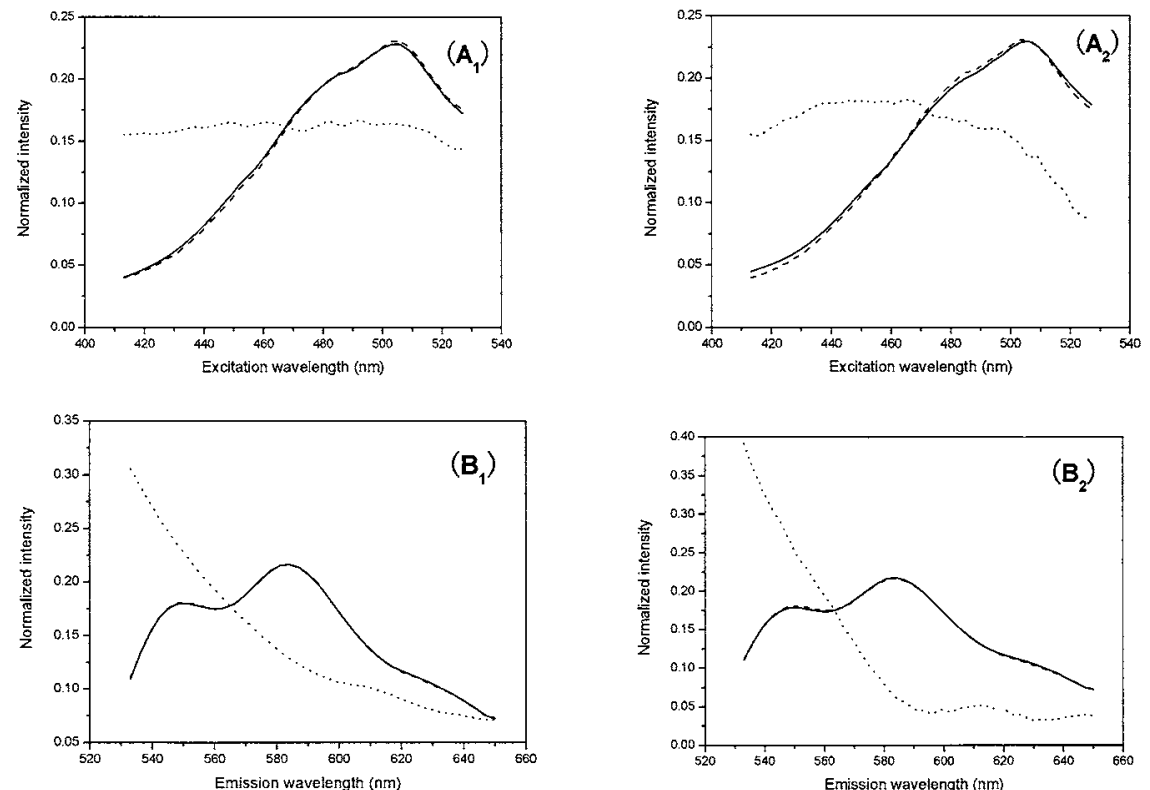

Fig. 3 Resolved (-) and actual (- - ) DNR and interferents (……..) excitation-emission spectral profiles in plasma using two algorithms when the chosen factor number was two: $\left(\mathrm{A}_{1}\right)$ and $\left(\mathrm{B}_{1}\right)$, PARAFAC; $\left(\mathrm{A}_{2}\right)$ and $\left(\mathrm{B}_{2}\right)$, AFR.

advantage. Both PARAFAC and AFR were able to produce acceptable results on plasma and urine samples. This is undoubtedly due to the presence of fluorescent plasma and urine components, whose influence has not been taken into account in the calibration set. These components not only exhibit emission intensities that overlap with the fluorescence signals from the analytes (Figs. 2A and B), but are also intrinsically variable and, thus difficult to model if the second-order advantage is not employed.

Two data arrays produced by fifteen mixture samples were 

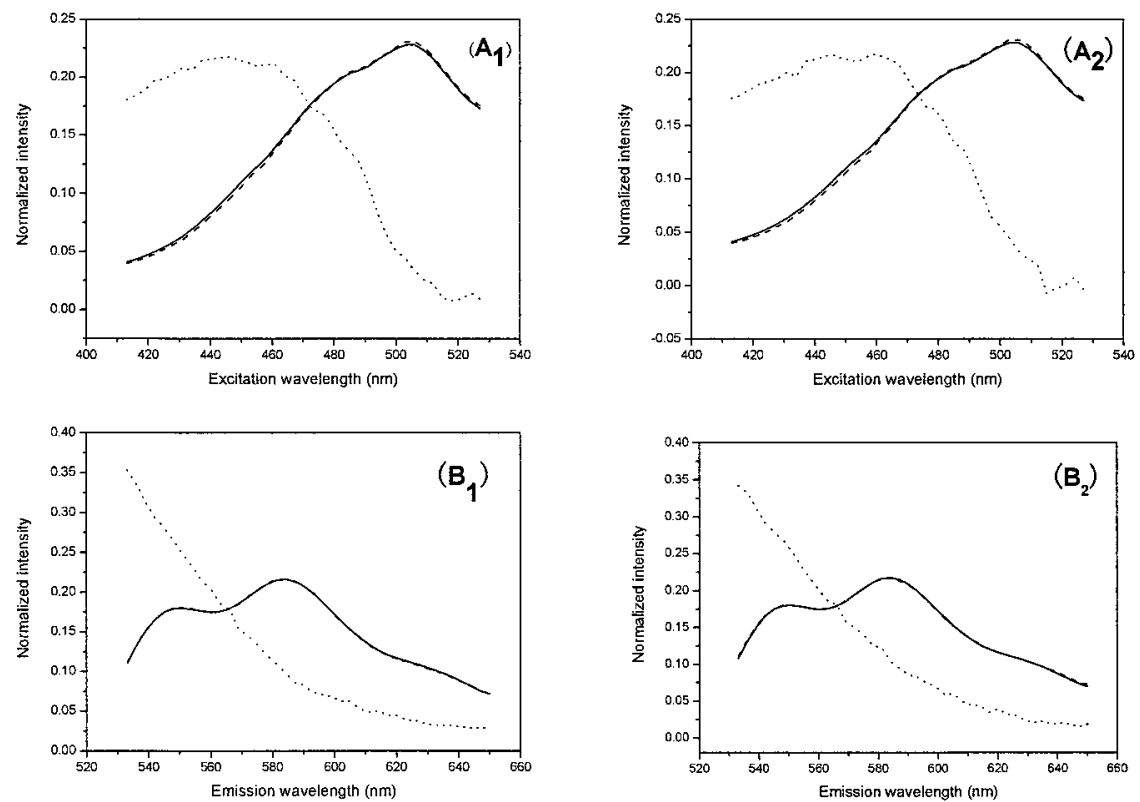

Fig. 4 Resolved (-) and actual (- -) DNR and interferents (…….) excitation-emission spectral profiles in urine using two algorithms when the chosen factor number was two: $\left(A_{1}\right)$ and $\left(\mathrm{B}_{1}\right)$, PARAFAC; $\left(\mathrm{A}_{2}\right)$ and $\left(\mathrm{B}_{2}\right)$, AFR.
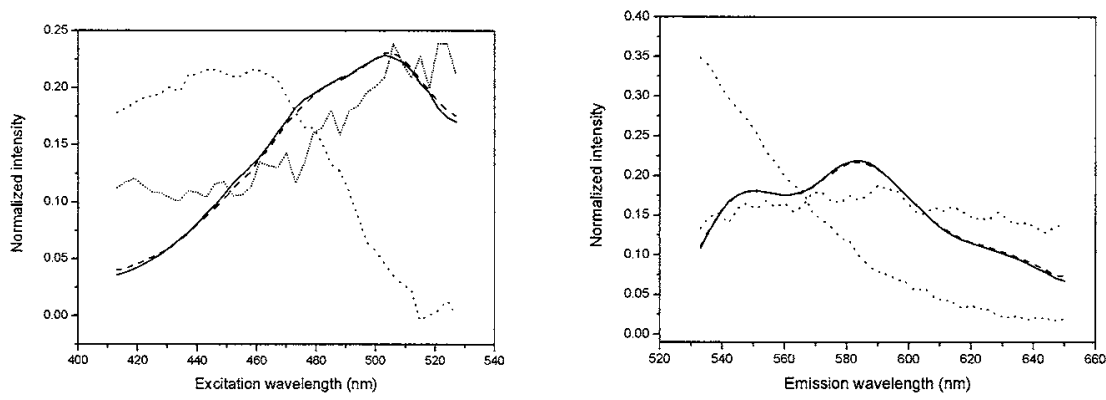

Fig. 5 Resolved (- $(-$ and actual (- - ) DNR and interferents (……...) excitation-emission spectral profiles in urine using AFR when the chosen factor number was three.

analyzed using the AFR and PARAFAC in order to resolve the actual profiles of the component. The first six samples were used as concentration-calibrating samples, and the remaining were used as concentration-predicting samples. The calibration samples with No. 7 - 11 samples compose one data array and the other with No. $12-15$.

Figures 3 and 4 show the resolved DNR excitation-emission spectral profiles together with the actual ones, respectively, in plasma and urine using the AFR and traditional PARAFAC algorithms when the chosen factor number was two. Figure 5 reveals the resolved DNR excitation-emission spectral profiles together with the actual ones in urine using AFR when the chosen factor number was three. It can be appreciated that the resolved DNR spectral profiles is nearly the same as the actual ones. Tables 2 and 3 display resolved concentrations of prediction samples and the figures of merit, respectively, in plasma and urine using AFR and PARAFAC. These results indicate that both algorithms can give satisfactory resolutions not only for excitation-emission spectral profiles and concentrations of prediction samples, but also for analytical figures of merit.

However, the results in Table 2 demonstrate that the performance of AFR surpasses that of PARAFAC in plasma. What is more, the results of the root-mean-squared error of prediction (RMSEP) and the concentrations of predictions samples $^{58}$ obtained using AFR when the chosen factor number was three exceeded that acquired using the traditional PARAFAC algorithm when the chosen factor number was two in urine. The reason for this is possibly that AFR can solve nonlinear constituents by fitting more factors. At the same time, it reveals the robustness of the estimated factor number using AFR.

\section{Conclusions}

A new algorithm, the AFR algorithm, has been developed as an iterative algorithm for the trilinear analysis of three-way data arrays. The results presented in this article demonstrate that the combination of fluorescence excitation-emission measurements and second-order calibration algorithms of three-way data is a powerful technique for the complex analysis of drugs in both plasma and urine, despite the serious interference from the plasma and urine background components. This is attributed to 
Table 2 Resolved concentrations of prediction samples and figures of merit in plasma using PARAFAC and AFR

\begin{tabular}{|c|c|c|c|c|c|c|c|c|c|}
\hline & \multicolumn{5}{|c|}{ Sample } & \multicolumn{4}{|c|}{ Figures of merit } \\
\hline & 7 & 8 & 9 & 10 & 11 & $\begin{array}{l}\text { RMSEP/ } \\
\mu \mathrm{g} \mathrm{ml}^{-1}\end{array}$ & $\begin{array}{l}\text { Sensitivity/ } \\
\text { AFU ml } \mu \mathrm{g}^{-1}\end{array}$ & Selectivity & $\begin{array}{c}\mathrm{LOD} / \\
\mu \mathrm{g} \mathrm{ml}^{-1}\end{array}$ \\
\hline Real & 0.6600 & 0.8800 & 1.1000 & 1.3200 & 1.5400 & & & & \\
\hline AFR & 0.5622 & 0.7902 & 0.9832 & 1.1255 & 1.3650 & 0.1412 & 1804.0 & 0.7525 & 0.0062 \\
\hline PARAFAC & 0.5082 & 0.7301 & 0.9209 & 1.0592 & 1.2931 & 0.2033 & 1511.0 & 0.6301 & 0.0059 \\
\hline
\end{tabular}

Table 3 Resolved concentrations of prediction samples and figures of merit in urine using PARAFAC and AFR

\begin{tabular}{|c|c|c|c|c|c|c|c|c|}
\hline & \multicolumn{4}{|c|}{ Sample } & \multicolumn{4}{|c|}{ Figures of merit } \\
\hline & 12 & 13 & 14 & 15 & $\begin{array}{l}\text { RMSEP/ } \\
\mu \mathrm{g} \mathrm{ml}^{-1}\end{array}$ & $\begin{array}{l}\text { Sensitivity/ } \\
\text { AFU ml } \mu \mathrm{g}^{-1}\end{array}$ & Selectivity & $\begin{array}{c}\mathrm{LOD} / \\
\mu \mathrm{g} \mathrm{ml}^{-1}\end{array}$ \\
\hline Real & 0.8800 & 1.1000 & 1.3200 & 1.5400 & & & & \\
\hline $\operatorname{AFR}(N=2)$ & 0.9985 & 1.2535 & 1.4392 & 1.6902 & 0.1364 & 2035.9 & 0.8469 & 0.0425 \\
\hline $\operatorname{AFR}(N=3)$ & 0.9811 & 1.2341 & 1.4102 & 1.6643 & 0.1138 & 708.5 & 0.3144 & 0.0505 \\
\hline PARAFAC & 0.9981 & 1.2534 & 1.4378 & 1.6881 & 0.1354 & 2029.3 & 0.8442 & 0.0446 \\
\hline
\end{tabular}

second-order advantage. Although the determination of DNR in human plasma and urine is satisfyingly successful, it is only a limited example of the enormous potentiality of these means in biomedical analytical fields, such as pharmacokinetic investigations in cancer patients. Further work is undoubtedly required to involve samples of higher complexity and to exploit more potentialities of the newly introduced algorithm.

\section{Acknowledgements}

The author gratefully acknowledges the support of the National Nature Science Foundation of China (Grant Nos. 20475014, 20375012 and 20435010) and the K. C. Wong Education Foundation, Hong Kong.

\section{References}

1. J. Robert, "A Clinician's Guide to Chemotherapy: Pharmacokinetics and Pharmacodynamics", 1998, Williams and Wilkins, Baltimore, 93.

2. A. Snycerski and Z. Fijalek, Chem. Anal. [Warsaw], 1996, 41,1025 .

3. S. Emara and I. Morita, Talanta, 1994, 41, 1973.

4. J. H. Miller, C. Pascal, and M. Tissieres, J. Chromatogr., 1987, 392, 361.

5. S. Fogli, R. Donesi, F. Innocenti, A. D. Paolo, G. Hocci, C. Harbara, and M. D. Tacca, Ther. Drug. Monit., 1999, 21, 367.

6. K. Fujiwara and N. Matsumoto, J. Immunol. Methods, 1990, 134, 227.

7. S. Sakura and H. Imai, Anal. Sci., 1985, 1, 413.

8. N. Simeon, E. Chatelut, P. Canal, M. Nertz, and F. Couderc, J. Chromatogr., A, 1999, 853, 449.

9. G. Hempel, P. S. Westhoff, S. Flege, and J. Boos, J. Chromatogr., B, 2001, 758, 221.

10. A. Kapur, J. L. Beck, and M. M. Sheil, Rapid Commun. Mass. Spectrom., 1999, 13, 2489.

11. K. S. Booksh and B. R. Kowalski, Anal. Chem., 1994, 66, $782 \mathrm{~A}$.

12. R. Bro, Chemom. Intell. Lab. Syst., 1997, 38, 149.
13. C. N. Ho, G. D. Christian, and E. R. Davidson, Anal. Chem., 1978, 50, 1108.

14. E. Sanchez and B. R. Kowalski, J. Chemom., 1988, 2, 265.

15. B. Wilson, E. Sanchez, and B. R. Kowalski, J. Chemom., 1989, 8, 493.

16. E. Sanchez and B. R. Kowalski, Anal. Chem., 1986, 58, 496.

17. K. Faber, A. Lorber, and B. R. Kowalski, J. Chemom., 1997, 11, 95 .

18. E. Sanchez and B. R. Kowalski, J. Chemom., 1990, 4, 29.

19. K. S. Booksh, Z. Lin, Z. Wang, and B. R. Kowalski, Anal. Chem., 1994, 66, 2561.

20. M. Gui, S. C. Rutan, and A. Agbodjan, Anal. Chem., 1995, 67, 3293.

21. M. Linder and R. Sundberg, Chemom. Intell. Lab. Syst., 1998, 42, 159.

22. M. Linder and R. Sundberg, J. Chemom., 2002, 16, 12.

23. P. J. Gemperline, K. H. Miller, T. L. West, J. E. Weinstein, J. G. Hamilton, and J. T. Bray, Anal. Chem., 1990, 64 , 523A.

24. A. K. Smilde and D. A. Doornbos, J. Chemom., 1991, 5, 345.

25. H. L. Wu, M. Shibukawa, and K. Oguma, J. Chemom., 1998, 12,1 .

26. B. C. Mitchell and D. S. Burdick, J. Chemom., 1994, 8, 155.

27. C. J. Appellof and E. R. Davidson, Anal. Chem., 1981, 53, 2053.

28. P. Geladi, Chemom. Intell. Lab. Syst., 1989, 7, 11.

29. H. A. L. Kiers and A. K. Smilde, J. Chemom., 1995, 9, 179.

30. P. Paatero, Chemom. Intell. Lab. Syst., 1997, 38, 223.

31. M. Linder and R. Sundberg, Chemom. Intell. Lab. Syst., 1998, 42, 159.

32. J. L. Beltran, J. Guiteras, and R. Ferrer, Anal. Chem., 1998, 70, 1949.

33. J. Z. Lu, H. L. Wu, J. H. Jiang, N. Long, C. Y. Mo, and R. Q. Yu, Anal. Sci., 2003, 19, 1037.

34. R. A. Harshman, UCLA Working Papers in Phonetics, 1970, 16, 1.

35. J. D. Carroll and J. J. Chang, Psychometrika, 1970, 35, 283.

36. Z. P. Chen, H. L. Wu, and R. Q. Yu, J. Chemom., 2001, 15, 439. 
37. Z. P. Chen, Y. Li, and R. Q. Yu, J. Chemom., 2001, 15, 149.

38. J. H. Jiang, H. L. Wu, Y. Li, and R. Q. Yu, J. Chemom., 1999, 13, 557.

39. J. H. Jiang, H. L. Wu, Y. Li, and R. Q. Yu, J. Chemom. 2000, 14, 15

40. Y. Li, J. H. Jiang, H. L. Wu, Z. P. Chen, and R. Q. Yu, Chemom. Intell. Lab. Syst., 2000, 52, 33.

41. R. D. Jiji, G. G. Andersson, and K. S. Booksh, J. Chemom., 2000, 14, 171.

42. R. Bro, Chemom. Intell. Lab. Syst., 1999, 46, 133.

43. J. A. Arancibia, A. C. Olivieri, and G. M. Escandar, Anal. Bioanal. Chem., 2002, 374, 451.

44. M. G. Trevisan, R. J. Poppi, Anal. Chim. Acta, 2003, 493, 69.

45. N. M. Faber, R. Bro, and P. K. Hopke, Chemom. Intell. Lab. Syst., 2003, 65, 119.

46. J. B. Kruskal, R. A. Harshman, and M. E. Lundy, in "Multiway Data Analysis", ed. R. Coppi and S. Bolasco, 1989, Elsevier, Amsterdam, 7.

47. R. Bro, "Muti-way Analysis in the Food Industry, Model,
Algorithms and Applications", Doctoral Thesis, University of Amsterdam, 1998.

48. A. Lorber, Anal. Chem., 1986, 58, 1167.

49. N. J. Messick, J. H. Kalivas, and P. M. Lang, Anal. Chem., 1996, 68, 1572

50. Y. Wang, O. S. Borgen, B. R. Kowalski, M. Gui, and F. Turecek, J. Chemom., 1993, 7, 117.

51. K. Faber, A. Lorber, and B. R. Kowalski, J. Chemom., 1997, 11, 419 .

52. C. N. Ho, G. D. Christian, and E. R. Davidson, Anal. Chem., 1980, 52, 1071.

53. N. M. Faber, J. Chemom., 2001, 15, 743.

54. A. C. Olivieri, Anal. Chem., 2005, 77, 4936.

55. A. C. Olivieri, A. A. Juan, A. M. de la Peña, D. M. Isabel, and E. M. Anunciacion, Anal. Chem., 2004, 76, 5657.

56. A. M. de la Peña, A. E. Mansilla, D. G. Gómez, A. C. Olivieri, and H. C. Goicoechea, Anal. Chem., 2003, 75, 2640.

57. A. C. Olivieri and N. M. Faber, Chemom. Intell. Lab. Syst., 2004, 70, 75.

58. K. Faber and B. R. Kowalski, Anal. Chem., 1997, 69, 1620. 